Johan Gøtzsche-Astrup \& Oluf Gøtzsche-Astrup Aarhus University, DK Denmark

\title{
Schemas of Contention: How Do the Public Interpret the Repertoire of Contention?
}

\begin{abstract}
The literature on contention conflates the structuring of contentious actions with their interpretation, obscuring the independent role of interpretation. We propose a schemas of contention theory that explicitly distinguishes between a schema of action and a schema of interpretation. Focusing on the latter, we theorize and study the public's interpretation of contention. In two studies of 1429 US citizens, we show that interpretation is structured by a schema that can be modelled in the dimensions of legitimacy and political legibility. Studies that neglect the schema of interpretation or reduce it to that of action misrepresent the structuring of contention.
\end{abstract}


Contentious politics is a central driver of social change in liberal democracies. Social actors use tactics such as public marching, pamphleteering, and even property destruction to put forward claims that challenge others or authorities (Tarrow 2011, p. 7). These tactics are often described through the dramaturgical metaphor of a "repertoire of contention," a stock of tactics that are used by contentious actors and recognized by the audience to whom they are directed (Tilly 1977; 1995; 2008). While the literature has assiduously mapped the use of these tactics, it has paid little attention to how they are interpreted by the audience to whom they are directed (Wouters 2019). The literature remains "movement-centric" (McAdam and Boudet 2012, p. 3). This is problematic because interpretation is central to the structuring of contention and its ability to bring about social change.

Take the recent Black Lives Matter protests and riots sparked by the police murder of George Floyd. People have marched in the streets and held public meetings but also looted, destroyed property, and attacked the police. While the interpretation of the former actions was mostly uncontested, it was more difficult for the public to ascribe meaning to the latter. They could be seen as political tactics that challenged the racialized exclusion of the protesters. They could also be seen as apolitical outbursts of social disorder. A gap opened up between the actions of the rioters and their interpretations by a public who struggled to ascribe political meaning to them. Ultimately, this put the rioters' ability to promote social change into question. This is just the most recent example of a recurring interpretative struggle about the meaning of riots and, more generally, contentious tactics (Body-Gendrot 2016).

The distinction between the actions and their interpretation, which became acute in the riots, holds for contention in general because meaning does not merely inhere in actions. It has to be ascribed to these through acts of interpretation (Taylor 1971). If we wish to understand the structuring of contention and its role in social change, we must theoretically and analytically distinguish contentious actions from their interpretation. Several research traditions have touched on the interpretation of contentious actions but have not tackled it directly. Framing theory in general and analyses of discursive opportunity structures in particular analyze the interpretation of contention but focus on contentious demands and neglect the actions themselves (e.g., Benford and Snow 2000; Ferree et al. 2002; Koopmans and Olzak 2004; Snow, Vliegenthart, and Ketelaars 2019). There is also a related literature on the media coverage of protest (e.g., Koopmans 2004; Seguin 2015; Andrews and Caren 2010; Vasi et al. 2015; Vliegenthart et al. 2016). However, although the media is an important gatekeeper of public 
discourse, we cannot extrapolate how people actually interpret contention from media coverage (Wouters 2019). There has recently been a welcome revival of interest in the public perceptions of protest. So far, this has been concerned with the narrow question of perceived legitimacy and favorability of particular protest forms and movements (e.g., Andrews, Beyerlein, and Tucker 2016; Park and Einwohner 2019; Zlobina and Vazquez 2018; Wouters 2019), missing other dimensions of interpretation. Moreover, it has grafted the analysis of interpretation onto a theory of contentious structures that remains movement-centric. Consequently, it has not developed a theory of contentious structure that incorporates the independent role of interpretation.

In this article, we enter into a dialogue with the repertoire of contention literature and develop a new schemas of contention theory. Drawing on Sewell (2005) and the sociology of culture and cognition (Lizardo 2017; Wood 2018), we theorize that contention is partly structured by two distinct schemas of action and interpretation. Focusing on the latter, we argue that the public, conceptualized as the general audience of citizens (Wouters 2019), interpret contentious actions through a set of embodied principles of interpretation that are transposable across contexts. We model this schema in the two dimensions of legitimacy and political legibility. Legitimacy concerns whether a tactics is seen as an acceptable action to carry out in society whereas legibility describes whether a tactic is recognisable as a political protest.

Based on this theory, we empirically analyze the structure of the public's interpretation of contention in the United States. In two survey studies of adult Americans, one of which is population representative, we empirically map the schema of interpretation in the dimensions of legitimacy and legibility. We ask respondents to imagine that a person has been shot and have them consider whether a series of actions taken in reaction to this shooting are legitimate and political. In the population-representative study, we also test whether the legitimacy and legibility of the actions depend on their context. We contextualize the shooting as either the result of a private or political disagreement and ask the respondents to consider whether the actions are legitimate and whether they are political. The results show a meaningful pattern in the interpretation of actions that is stable even across contexts. They also reveal that people distinguish between the legitimacy and political legibility of actions. We therefore conclude that there is a schema of interpretation that structures the ascription of meaning to contentious actions and that it can be modelled through the dimensions of legitimacy and legibility. 
In putting forward and applying the schemas of contention theory, the article contributes in three ways to the extant literature on contention. First, the schemas of contention theory clarifies the embodied structures of contention. This counters the movement-centrism of the repertoire of contention literature and gives a solid theoretical footing to the burgeoning literature on the public perception of protest. Second, it provides a fine-grained and systematic empirical mapping of how the public make sense of contention in the United States. Given the centrality of contention to contemporary society, understanding how it is ascribed meaning is important in its own right. Third, the schemas of contention theory and the analysis reveal a novel research agenda concerned with the embodied structure of contention, which we unfold in the discussion.

\section{Structuring Contention in the Extant Literature}

We are fundamentally interested in the structure of contention. More specifically, the structuring of the public's interpretation of contentious actions. While the extant literature on the public perception of protest has not engaged clearly with this problem of structure, it has partly been dealt with under the heading of repertoires of contention. The repertoire describes what actions can be used and recognized as contentious in a given time and place. Below, we therefore first review the extant work on repertoires of contention before arguing that we need to reconceptualize it by distinguishing between schemas of action and interpretation.

\section{The Structure of Contentious Action}

In setting out the notion of a repertoire, Tilly (1977, p. 493) sketched two research agendas for the mapping of contention, which the literature has since followed. Analyzing the repertoire means tracing the evolution of individual forms of contentious actions and of repertoires as such. Thus, Traugott (1993) traces the evolution of the barricade, Biggs (2013) the form of suicide protests, Wei (2015) the creation of street behavioral art, and McCurdy and colleagues (2016) the role of protest camps. It is, however, the analysis of the repertoire as such that has drawn the most attention. Here, the focus has been on the shift from a "traditional" repertoire to a "modern" one (Tarrow 2011, pp. 41-2; Tilly 1995, p. 46; Wada 2012). The traditional repertoire consisted of forms such as rough music, tarring and feathering, and grain seizures. In contrast, the modern repertoire is exemplified by public meetings, mass demonstration, and pamphleteering.

These analyses assume that the repertoire of contention is meaningful for the actors that draw on it (Ring-Ramirez, Reynolds-Stenson, and Earl 2014; Tarrow 2011, p. 51). This dimension of meaning 
is usually understood through the metaphor of a toolbox from which actors choose appropriate forms of contention (Swidler 1986, p. 277). Cultural scholars have added to this approach by analyzing how particular agents deploy tactics and invest them with different meanings (Doherty and Hayes 2019; Gillan 2020; Goodwin and Jasper 1999). While these analyses are therefore interested in the structuring of meaning, they have focused on the perspective of the contentious actors themselves and not the public's interpretation of these actions. They remain movement-centric (McAdam and Boudet 2012, p. 3). In doing so, we argue that they implicitly assume that the structuring of interpretation is homologous to that of action. In making this assumption explicit, we also suggest that it misrepresents how contention is actually structured.

\section{The Independent Role of Interpretation}

Acting contentiously is a form of communicative action (Larson 2013, p. 870). Contentious actors usually wish to make themselves understood by an audience (Andrews, Beyerlein, and Tucker Farnum 2016; Wouters 2019), which may ultimately be the public. Even prefigurative direct action, which constructs alternatives to the existing social order, involves the communication of alternatives to an audience (Yates 2015, p. 19). In order to be understood, actors articulate their challenges by using tactics that the audience can decode. The necessity of interpretation is implicitly acknowledged in the repertoire literature. For example, Tarrow (2011, p. 39) notes that repertoires consist of what people do, know how to do, and what others expect them to do. While the first two statements concern contentious actions, the final one is clearly about their interpretation. In effect, the literature already assumes the existence of a structure of interpretation through which the public can make sense of contention. We are making explicit this assumption.

However, once we have to deal explicitly with the structuring of interpretation, we must confront the question of how action and interpretation relate to each other. Because the literature has not raised this question, it has implicitly assumed a homology between them. In Tarrow's terms, once we have mapped what people do and know what to do, the expectations of others follow automatically.

There are two problems with the assumption of homology. First, because the consequences of contentious actions depend on their interpretation by a wider audience, we must analyze this interpretation in its own right. We must allow for the very real possibility of misunderstanding and failed communication, as the Black Lives Matter contention shows. If we do not, we risk misrepresenting how contention is actually structured. Second, the homology obscures a fruitful line of research. 
Johan Gøtzsche-Astrup \& Oluf Gøtzsche-Astrup

Aarhus University, DK Denmark

Namely, how do the public distinguish contentious actions from each other? Why are certain actions seen as acceptable protests while others are not? In short, how do the public make sense of contention? It is only by allowing for an asymmetry in the structuring of action and interpretation that these questions can be posed and answered.

\section{The Schemas of Contention Theory}

The problems can be overcome by reconceptualising the repertoire of contention to incorporate the independent role of interpretation. We do so by positing a schemas of contention theory. We argue that the repertoire of contention consists of two embodied schemas, that of action and that of interpretation. Honing in on the schema of interpretation, we model it in the two dimensions of legitimacy and legibility.

\section{Schemas of Action and Interpretation}

In conceptualizing schemas, we draw on both Sewell's inflection of Giddens' (1984) structuration theory and recent developments in the sociology of culture and cognition. For Sewell (2005, pp. 12932), schemas are the rules of social life, principles that pattern social practices. They are structural in the sense that they are transposable, applicable across different contexts. Recently, Sewell's schemas have come under critique for their unclear ontological status. Sewell follows Giddens in casting them as "virtual," a somewhat vague notion. Drawing on Bourdieu's (1990) sociology of practice and on cognitive theories, the sociology of culture and cognition has instead stressed the embodied nature of schemas (e,g., Lizardo 2010; 2017; Wood et al. 2018). On the most recent account, schemas are a form of non-declarative personal culture. They are personal because they are internalized in individuals and not externalized through public symbols, discourses, or institutions. They are non-declarative because they are acquired through slow processes of enculturation that form practical knowledge and knowhow that may not be immediately accessible to the individual. This contrast between the non-declarative form and its declarative counterpart is drawn directly from the distinction between two different modes of cognitive processing (Lizardo 2017; Wood et al. 2018).

In our schemas of contention theory, we strike a balance between Sewell's formulation and the culture and cognition tradition. We appreciate the latter's distinction between personal and public culture because it makes the ontological status of schemas clear. Furthermore, it underscores that the structure of contention cannot be reduced to embodied schemas although they form an important part of it. In proposing a schemas of contention theory, we are therefore not arguing that it describes 
the structure of contention tout court but rather the embodied part of this structure. We also acknowledge the distinction between declarative and non-declarative personal culture. However, we agree with Kurakin (2020, p. 80) that it is not apparent whether this distinction can be directly translated from the cognitive sciences to sociology, that is, whether the structures of cognition necessarily align themselves with the structures of personal or embodied culture. We therefore choose to soften the distinction between declarative and non-declarative personal culture. We see declarative and nondeclarative personal culture as describing differences in what Sewell (2005, p. 146) calls the depth of schemas, the degree to which they are foundational to the practices they structure and taken for granted by the individuals in which they are embodied. This does not exclude the possibility of two distinct forms of personal culture but remains open to other ways of conceptualizing it. In this article, we therefore conceptualize schemas as transposable principles of personal culture that individuals employ to make sense of and act in the social world. We draw the stress on transposability from Sewell and the schemas' personal quality from the sociology on culture and cognition.

More specifically, we theorize that the structure of contention consists of two such schemas: first, a schema of action, which is the one that has been studied in the contention literature. It governs the actions available to contentious actors, including the meaning, identities, and emotions that are intertwined with the actions (cf. Doherty and Hayes 2019; Flesher Fominaya 2015; Ring-Ramirez et al. 2014). The second is a schema of interpretation. It governs the ways in which an audience, in this case the public, ascribes meaning to the actions. This second schema has been subsumed under that of action. While this may be defensible in the case of seasoned activists who use their own contention to interpret that of others, it is not for the public at large.

In speaking of a schema of interpretation, then, we focus on the embodied and transposable principles that individuals use to interpret contention and which produce a pattern in this interpretation. While framing (e.g., Benford and Snow 2000; Snow et al. 2019) may influence the interpretation of particular contentious actions, it evokes and is constrained by this schema (Wood et al. 2018). Finally, we follow Wang and Soule (2016, p. 524) in seeing actions (or acts) and tactics as synonymous and distinguish them from the higher-level "forms" such as rioting and demonstrating. While these forms are undoubtedly important (Tilly 2008, p. 17), we focus on actions because they are the concrete manifestations of contention that the public interpret (cf. Hall, Rodeghier, and Useem 1986, p. 564). 
We model the schema of interpretation in two dimensions. To be clear, there are many extant principles of categorization. Contentious tactics can be "contained" or "transgressive" (McAdam, Tarrow, and Tilly 2001); "contained", "disruptive," or "“violent” (Tarrow 2011); "modular" or not (Wada 2012); "rigid" or "flexible" (Wada 2016). All of these are designed as analytical tools through which the researcher categorizes different tactics of contention. For example, McAdam, Tarrow, and Tilly (2001, p. 7) distinguish contained from transgressive contention in order to analyze transitions between them and the effects of contention on social change. They themselves categorize contentious tactics as either one or the other and use these divisions to analyze the causal processes of contention. While the two dimensions of legitimacy and legibility have certain overlaps with such distinctions, their rationale is fundamentally different. We do not use them to categorize different contentious actions ourselves but to analyze how actors interpret these.

We stress that the dimensions do not exhaust the complexity of interpretation. They are a theoretical model of the schema of interpretation whose practical logic necessarily makes them fuzzier than the dimensions suggest (Bourdieu 1990, p. 81; Lizardo 2010). However, we do expect the dimensions to capture some fundamental aspects of the schema. Faced with a contentious event, an observer makes two fundamental interpretations. First, whether the actions employed are legitimate and, second, whether they can be characterized as political at all. If individuals did not distinguish between the legitimacy and legibility of actions, it would be difficult to make sense of contention in the first place.

First, contentious actions can be seen as more or less legitimate. By legitimacy we mean actions that are acceptable to carry out in society. When individuals orient themselves socially, they do so partly by drawing moral boundaries between what is acceptable and what is not (Lamont and Molnár 2002). This is also the case in the realm of politics (Tarrow 2011, p. 110). As Alexander (2006) has argued, the distinction is not merely a legal rule but a part of the public culture. We argue that it is also a part of the personal culture of individuals. It is this dimension that the literature on the public perception of protest has been concerned with (e.g., Andrews et al. 2016; Park and Einwohner 2019; for a set of classical studies on legitimacy perceptions see Hall, Rodeghier, and Useem. 1986; Olsen 1968; Rodeghier, Hall, and Useem 1991).

Second, an action can be more or less legible as political. Indeed, this distinction makes it possible to speak of a repertoire of contention at all, because there must be a distinction between those actions that challenge the social order and those that do not. Turner (1969), reflecting on the US riots in the 1960s, made a similar point in arguing that actions must correspond with a "folk concept" of protest 
in order to be classified as such. Although the subsequent literature has not completely neglected the dimension of legibility (Jeffries, Turner, and Morris 1971; Tarrow 1993a, p. 71), it has tended to either conflate it with questions of legitimacy or gloss over it completely. As the debate on the political or apolitical nature of the recent Black Lives Matter riots shows, this dimension should be taken seriously and analyzed in its own right if we are to understand how the public's interpretation of contention is structured. Looting and property destruction may not only be less legitimate but also less politically legible compared to picketing or pamphleteering. We note that in speaking of legibility, we refer to whether the contentious tactics are understood as political protests. While there may be a distinction between a broad notion of contentious tactics (McAdam et al. 2001; Tarrow 2011, p. 7; Tilly 2008, p. 5) and a narrow one of protest tactics, the two terms are usually treated as synonymous in the repertoire literature (e.g., Taylor et al. 2009; Wang and Soule 2016). Most importantly for us, the meaning of "protest" in ordinary language is likely synonymous with a broad definition of contention as calls for social change that challenge others or authorities.

Our theoretical argument can be summarized in three points. First, we need to reconceptualize the repertoire of contention to incorporate the independent role of interpretation in the structuring of contention. Our schemas of contention theory is composed of embodied schemas of action and interpretation. Second, both schemas are structurally rooted. In the case of the schema of interpretation, the interpretation of contentious actions is patterned and transposable across contexts. Third, we can analyze this schema empirically by modelling two primary dimensions of interpretation: legibility and legitimacy.

These points result in three empirical expectations. First, if we can model the schemas through the dimensions of legibility and legitimacy, we should observe substantial and significant differences between the two dimensions. Second, if the schemas of contention are structurally rooted, we should be able to map out a meaningful pattern in our data using the two dimensions. Third, the structure of the schemas also means that they are transposable. Therefore, we should not find overwhelming differences in legitimacy and legibility in different contexts. In other words, the meaningful pattern ought to be relatively stable across contexts. The schemas of contention theory is fruitful if it allows us to observe a stable pattern in the interpretation of tactics with variation in and between the dimensions.

\section{Empirical Strategy}


We test these expectations in two survey studies of the American population. We use panel participants on crowdsourcing marketplaces, which has the advantage of reaching our target population of ordinary individuals not particularly interested in politics or protest. Furthermore, these platforms have the advantage of large panel pools less accustomed to political issues than what studies of, for example, social science students provide, giving us the opportunity to reach more naïve participants. Finally, conducting the studies through crowdsourcing marketplaces enables direct payment of participants, ensuring a sustainable and socially responsible use of participants (Robinson et al. 2019).Next, we discuss how we measure the schema of interpretation before turning to the individual studies.

\section{Measuring the Schema of Interpretation}

We can distinguish two tasks in testing the schemas of contention theory: first, what tactics to consider, and second, how to distinguish between the legibility and legitimacy of the tactics.

To build a repertoire of tactics, we started with the Dynamics of Collective Action (DOC) dataset ${ }^{1}$, which contains more than 23,000 New York Times articles covering protest events in the United States between 1960 and 1995. To be included in our study, the event had to have been collective, taken place in public, been a "protest" event, and put forward a claim or grievance. Events that involved racial or ethnic violence were also counted even if they did not include an explicit claim. Scholars employed on the project content-coded information on each event, with intercoder reliability above 90 percent for events (Wang and Soule 2016). Although selection and coverage bias may exist, meaning that the dataset does not provide a complete list of possible protest events, it is not problematic for our purposes. However, we do assume that the events cover the main tactics in the repertoire.

There are several advantages of proceeding from this database. First, as the events are all reported in the news media, it represents the way most ordinary people would encounter a specific protest event (Koopmans 2004). Second, the events have been coded into the level of tactics, which is the level of analysis that we are interested in (Wang and Soule 2016). Third, each tactic was arguably political—and likely legitimate — from the perspective of the contentious actors themselves. Variation in the interpretation of tactics therefore provides a strong argument against the homology between the schemas of action and interpretation and for the independent analysis of the latter, although we do not test this homology directly.

\footnotetext{
${ }^{1}$ See http://www.dynamicsofcollectiveaction.com/ for more in-depth documentation and discussion of this dataset, including a definition of protest events.
} 
In operationalizing the tactics, we therefore adapted Wang and Soule's (2016, p. 542) list of 57 tactics. We elected to combine some of the tactics (e.g., combining tactic 10 "Film showing" and tactic 16 "Photo exhibiting" into the tactic "Hosting an art exhibition"), and removed a few others (e.g., "Bed racing", which refers to a very specific historical issue). As the dataset only included tactics up until 1995, we included a few modern tactics (e.g., "Posting opinions on social media"). Thus, we ended up with 41 tactics, as represented in Table 1. Further reduction in the number of tactics risked overinclusive categories and a loss of detail in our mapping. To lessen the cognitive load placed on participants, and to increase the resolution of our map, we opted for a slider format rather than Likertstyle response categories. Appendix A table A1 provides a tactic-to-tactic comparison of the 41 tactics and Wang and Soule's 57 tactics.

We then needed to measure how each tactic scored on the dimensions of legibility and legitimacy. As tactics are never performed in a vacuum, we wanted to present participants with an event that could meaningfully result in the array of tactics under analysis. We decided that the shooting to death of an individual would be both recognizable to the participants and could result in the tactics. We operationalized the legibility of tactics by asking participants to indicate their opinion on whether each tactic, presented as a possible reaction to the shooting, was a political protest. They could grade the tactics from a scale of 0 , indicating that it was surely not a political protest, to 100 , indicating that it was surely a political protest. We operationalized the legitimacy dimension in a similar manner by asking people to indicate their opinion on whether each tactic was an acceptable reaction to the shooting. We used a scale ranging from 0 , indicating that it was surely not acceptable, to 100 , indicating that it was surely acceptable. The tactics were presented in a randomized order so as to avoid order effects. We report instructions for participants and precise wordings of questions in Appendix A.

--- [INSERT TABLE 1 HERE] ---

Conducting the Studies and Demographics ${ }^{2}$

\footnotetext{
${ }^{2}$ All data used in this manuscript is made available at https://osf.io/hr37c/
} 
Both studies received institutional review board approval prior to fielding, and all participants read and agreed to a consent form informing them of their rights prior to starting the survey.

In the first study, we wanted to investigate our expectation that if there were a schema of interpretation, then we ought to find substantial independent variation between the two dimensions and a meaningful pattern to how people interpret the tactics. Between October 15 and 17, 2019, 396 respondents completed the first survey.

In the second study we attempted to replicate and extend the findings from the first study in a representative sample of the American population. We also wanted to further investigate the structure of the schema by testing whether it was transposable across contexts. We varied the political nature of the context, reasoning that this would be a critical test of our expectation. If the tactics were interpreted in similar ways in private as well as political contexts, it would be strong evidence for the existence of a structural schema of interpretation. One-third of the respondents were therefore asked to consider the fatal shooting of an individual as a result of a private quarrel, while another third were asked to consider the same shooting as a result of a political quarrel. The last third of respondents were not given any specific context for the shooting. We collected the data to be representative of the adult US population for age in decades, gender, race, and education. Between November 19 and 22, 1033 respondents completed the second questionnaire.

The distributions on key observable demographic outcomes are reported in Appendix B1. Overall, both samples match up well with the target population on demographics (age, sex, race) and socioeconomic variables (income, education). The samples were not selected based on political variables. In study 1 , but not in study 2, Democrats seem to be oversampled. About one-tenth of respondents indicate having participated in a protest during the last year, and between four-fifths (study 1) and two-thirds (study 2) report having voted in the last election where they were eligible to vote.

\section{Schemas of Interpretation in the US Public}

\section{Differences between Dimensions}

Our first empirical expectation was that if we can model the schema through the dimensions of legibility and legitimacy, we should observe substantial and significant differences between the two dimensions. In the first study, the means for legibility and legitimacy for all tactics differed statistically significantly $(\phi<.05)$ from each other. More than half $(21)$ differed a quarter of the scale or more 
(absolute differences at or above 25 points), and five tactics differed by more than half the scale length. Using the means for each tactic on the two dimensions as observations in a regression, the means for each tactic in the two dimensions correlated positively in the first study, but not strongly $(r=.346, p$ $=.027$ ) , each dimension explaining $9.68 \%$ of the variation in the other. These results support the expectation that individuals do distinguish between legitimacy and legibility in their interpretation of tactics. The means and standard deviations, as well as the absolute differences and results of simple $t$ tests for differences for the first study are reported in Appendix table C1. However, since the sample was not representative of the broader American population, we should be careful in extrapolating results.

The nationally representative sample strengthens the external validity of our claim. As in the first study, we used a two-sample $t$-test to explore if the means for each tactic differed in terms of their legitimacy and legibility. Table 2 reports the sample means and standard deviations for each tactic in each dimension, as well as the absolute differences. For 37 of the 41 tactics, the difference was significant at a very low alpha level $(p<.001)$. One tactic (petitioning) was only significant at the .05 level, and three tactics did not differ in terms of their legitimacy and legibility (setting off fireworks, holding signs in public, and recruiting). Thirty tactics had absolute legitimacy-legibility differences of more than ten points on the 0-100 scale, and eight tactics had differences of more than 25 points. There was a stronger legitimacy-legibility correlation than for the first study $(r=.479, \mathrm{p}=.002)$. The correlation does not reach the conventional level for a large correlation of $r>$. The variation in each dimension explained $21.0 \%$ of the variation of the other. In other words, knowing the exact variation in the tactics on one dimension, we can explain little more than one-fifth of the variation in the other dimension. About four-fifths of the variation in one dimension therefore cannot be explained by the other, which speaks against legitimacy and legibility being identical dimensions.

\section{--- [INSERT TABLE 2 HERE] ---}

These results support our claim that we can model the schema of interpretation through the dimensions of legitimacy and legibility. That is, the American public do actually distinguish between legitimacy and legibility when ascribing meaning to tactics. The results make a substantial difference in how we should understand the public's interpretation of individual tactics. For example, whereas 
blockading roads is generally seen to be moderately political, with a mean score of $53.2(\mathrm{SD}=38.5)$, it is generally seen as strongly illegitimate, with a mean legitimacy score of $25.6(\mathrm{SD}=32.0)$. Contrast this with the act of lighting candles, which is somewhat politically illegible (mean $=43.9, \mathrm{SD}=36.7$ ) but much more legitimate (mean $=79.4, \mathrm{SD}=27.5$ ) than blockading roads. These tactics are clearly categorically different. However, because the extant theoretical approaches tend to conflate legitimacy and legibility (e.g., McAdam et al. 2001; Tarrow 2011), they would struggle to make sense of this difference. Our modelling of dimensions captures an important and hitherto neglected distinction in the way that the public ascribe meaning to contentious tactics.

The relatively large variance in responses across individuals, as indicated by the large standard deviations, was an unexpected result. We suggest two interpretations of this. First, as an artefact of asking respondents to respond using sliders means selecting the extreme responses of 0 and 100 (as an indication of completely acceptable or unacceptable conversely legible or illegible) creates large variation in itself. Second, the large variance could be a real reflection of political and sociodemographic differences in the public. We take up this last interpretation in our discussion of group differences.

\section{Mapping the Schema of Interpretation}

Our second expectation was that if the schemas are structurally rooted, we should be able to map out a meaningful pattern in our data using the two dimensions. We investigate this by graphically mapping the schema in the two dimensions.

While the large differences in dimensions already indicate a pattern in the interpretation of tactics, we also mapped this pattern graphically to see whether it was actually meaningful. Figure 1 plots the means for each tactic in a graph of the protest dimensions for the nationally representative sample. It shows large variation in tactic evaluations, with four quadrants emerging. This result replicates the results from the first study (reported in Appendix figure C1). We briefly lay out the quadrants.

The quadrant defined by high legibility and high legitimacy scores above the midline of the scale and contains conventional tactics such as the petition, marching in the streets, distributing leaflets, and recruiting individuals to join political organizations. The borders of this quadrant are defined by tactics that are more legible than legitimate (the walkout), and more legitimate than legible (social media activity). These tactics seem to belong to a set of conventional protest tactics that are staple features of American political society. In Tilly's (2008, p. 145) terms, they are a part of the "super-strong" social movement repertoire. 
The quadrant defined by low perceived legibility and low legitimacy contains two clusters: first, a small group of tactics seen as somewhat illegitimate and illegible (fireworks, selling items, and making loud noises in the street); second, a relatively large cluster of illegible and extremely illegitimate tactics, which include looting, verbal attack, physical attack, hostage taking, and damaging property. These tactics include actual or potential violence and are the ones usually associated with riots, such as those following the police murder of George Floyd. Dancing sits as an isolated tactic with some legitimacy but the lowest legibility.

\section{--- [INSERT FIGURE 1 HERE] ---}

Below the midline on legibility and above the midline for legitimacy, we find tactics that are seen as legitimate but not as easily decodable as political protests. These tactics include highly legitimate tactics such as the silent vigil, meditation, worshipping, and lighting candles, and a cluster of somewhat legitimate but less legible tactics such as holding an art performance or exhibition, or ringing bells. They are probably associated with religious or artistic practices that are not clearly political.

The final quadrant contains five tactics perceived as illegitimate but still legible as political protest. These include the somewhat illegitimate actions of shouting slogans and going on hunger strike, and very illegitimate but nevertheless legible actions of disrupting meetings, creating blockades, and building takeovers.

The emergence of a meaningful map of the schema of interpretation and four distinct quadrants support our second empirical expectation of a meaningful pattern. We take this as strong evidence for our claim that there is a schema that structures the interpretation of contention.

\section{The Transposability of the Schema}

Our third expectation was that if the schema is transposable across contexts, as we suggest, the meaningful pattern observed above ought to be relatively stable across contexts. In the nationally representative sample, as in study 1, participants were presented with a case of someone who had been shot to death, and evaluated the tactics resulting from this shooting. However, we experimentally varied the context of the shooting. Each respondent was randomly allocated to one of three condi- 
tions. In the first condition, seen by one-third of the sample, participants were presented with a context-free shooting identical to study 1. For the other two-thirds of participants, however, we provided one of two contrasting contexts for the shooting. In one context, the shooting was privately motivated and in the other, the shooting was between political adversaries. When comparing these two conditions to each other, we should be able to gauge the context dependency of the schema of interpretation.

The results indicate that the pattern is stable across the contexts. Appendix tables D1 and D2 and Figure D1 report the results of tests comparing the means in the private and political contexts. Overall, context did little to change most evaluations of legitimacy and legibility, and the differences that we found were small. The difference in legitimacy across the contexts was statistically significant for seven tactics: collecting signatures for a petition, marching, selling items, lobbying politicians, conducting an opinion poll, meeting politicians, and conducting a sit-in. The largest point difference was for meeting with politicians at 7.6 scale points. For legibility as well, seven tactics differed across conditions. These were lighting candles, holding a silent vigil, worshipping, laying down wreaths, holding an art performance, posting on social media, and filing a lawsuit. The largest point difference was for the silent vigil at 7.57 scale points. In all cases, tactics were interpreted as more legitimate and more legible in the political context than in the private context.

\section{--- [INSERT FIGURE 2 HERE] ---}

When compared to the differences across previous protesters and non-protesters (Appendix tables E1 to E8), there are both fewer impacts of context, and the observed differences are much smaller in scale points. If we plot the means for each of the 14 tactics that differed on at least one dimension between contexts (Figure 2), it becomes apparent that each tactic seems anchored around a particular position in the two dimensions. While context does have an effect on the interpretation of some tactics, it is small and does not challenge the transposability of the schema. Having a public discussion is seen as political and legitimate no matter the context. Likewise, looting or property destruction are neither legitimate nor clearly political actions even when they are a response to a politically motivated killing. In ascribing meaning to protest actions, the public seem to draw on both contextual clues and their underlying schema of interpretation. In other words, we do not deny the effect of framing on 
the interpretation of particular contentious tactics (Snow et al. 2019) but argue that we have uncovered a schema that constrains the effects of this framing. If we wish to understand how the public make sense of contention, we should not just study how individual tactics are framed and interpreted by the public but should also analyze the schema of interpretation that structures this interpretation.

Overall, our results reveal a stable pattern to the interpretation of contention, which can be mapped through the two dimensions of legitimacy and legibility. They support our schemas of contention theory, which distinguishes between two aspects of personal contentious culture: schemas of action and schemas of interpretation. The results indicate that the latter is structural because it is transposable across contexts and can be modelled in the dimensions of legitimacy and legibility.

\section{Developing the Schemas of Contention Theory}

The schemas of contention theory allows us to uncover a hitherto neglected element in the embodied structure of contention. While the results speak to the fruitfulness of this theory, they also raise a series of questions about the transposability of the schema of interpretation, group differences in interpretation, and its openness to change. We discuss these in turn before examining how the schemas of contention theory gives us a new perspective on contention's ability to bring about social change. We conclude by outlining the limitations of the studies.

\section{The Transposability of the Schema of Interpretation}

The results support our claim that there is a schema that structures the interpretation of contention in the American public and that it is transposable across different contexts. However, one could ask what would happen if the context were fleshed out even further. In thinking about this problem, it is important to distinguish between the dimensions of legitimacy and legibility. In the case of legitimacy, we can supplement our results with the budding literature on the public perception of protest. It supports our claim that the ascription of legitimacy is relatively stable. Wouters (2019) found that a particular set of tactics that display worthiness, operationalized as the absence of disruption, consistently heightened the audience's support for protests. He replicated this pattern in the very different contexts of a Black Lives Matter protest in the United States and an asylum seeker protest in Belgium. Simpson, Willer, and Feinberg's (2018) study of the negative effects of violence on protest support also suggest a degree of stability across contexts. Although support should not be equated with legitimacy, the results do add further plausibility to the transposability of the schema of interpretation. 
Turning to the dimension of legibility, our results indicate that legibility is as transposable across context as legitimacy. However, as we note earlier, this dimension has been more or less neglected in the literature, and the discussions that do exist are either theoretical or methodologically flawed (Turner 1969; Jeffries et al. 1971). We therefore need further empirical studies of the legibility of tactics in different contexts. One key distinction that these studies may make is between two types of illegibility. Some tactics are illegible quite simply because they are unfamiliar to audiences as a protest tactic. In fleshing out the context even more than we have done, one might expect that the audience could see the particular manifestation of an unfamiliar illegible tactic as political. Art exhibitions may fall in this category. Other tactics are illegible because they are defined in opposition to legible tactics and not because they are unfamiliar to the audience. For example, looting and property destruction are often contrasted to protest tactics such as marching in the streets and pamphleteering. We have seen this most recently in the case of the Black Lives Matter movement where both opponents and supporters of the movement tried to draw precisely this distinction. In this case, the tactics' oppositional illegibility reaches deep and is difficult to overcome because it helps to constitute the schema of interpretation itself. If these tactics are to be interpreted as protests, it may require an overall shift in the understanding of protest.

\section{Group Differences}

In this article, we focus on the schema of interpretation in the general American public. However, there are also important group differences in the interpretation of protest (e.g., Hall et al. 1986; Park and Einwohner 2019; Rodeghier et al. 1991). Although we do not pursue this line of questioning in the article, the schemas of contention theory is able to shine a new light on these differences by nuancing group differences in legitimacy and distinguishing them from differences in legibility. We demonstrate this in Appendix tables E1-E8 where we give an overview of the differences in legitimacy and legibility ratings across political, socioeconomic, and demographic characteristics.

The pattern tallies with previous research on group differences in legitimacy ascriptions. Education, previous protest experience, democratic partisan identity, and identifying as a male are related to higher legitimacy ratings. Those who describe themselves as black also had higher legitimacy ratings than those who described themselves as white. Our finely-grained image of the different protest tactics allows us to further nuance this general picture. For example, while previous protest experience relates to higher legitimacy ratings for almost all tactics (38 out of 41), higher education was only related to higher ratings for nonviolent tactics (cf. Hall et al. 1986; Park and Einwohner 2019). Identifying as 
black, on the contrary, was related to higher acceptance of classical protest tactics and disruptive and violent tactics such as blockading or looting. Although this does not mean that black people accept violence to a higher degree (cf. Rodeghier et al. 1991), it means that they generally saw it as much more legitimate than self-identified white people. These differences suggest that previous protest experience, education, and being black shape the interpretation of protest in substantially different ways. This is obscured in analyses that use a less finely detailed measure of protest tactics.

The group differences in the dimension of legitimacy did not necessarily replicate themselves in the dimension of legibility. Take the example of partisan identity. Republicans indicated significantly lower legitimacy ratings than Democrats on 27 tactics. However, this difference was attenuated in the dimension of legibility. Here, only 12 tactics were significantly less legible for Republicans than for Democrats and the correlation coefficients were smaller than for legitimacy. The differences for education, gender, and age were similarly attenuated. Moreover, the group legibility differences for race were not the same as in the dimension of legitimacy. Most notably, self-identified black people were not more likely to see violent tactics as protests although they were more likely to accept them than self-identified white people. The results suggest that the ascription of what counts as protest may be more widely shared than the ascription of what counts as legitimate. That is, individuals are more likely to differ on whether a particular action is acceptable than they are on whether it is a protest. Public disagreement about the acceptability of protest can coexist with agreement on the meaning of protest and political participation more generally. It is only because our schemas of contention theory allows us to distinguish between legitimacy and legibility that we are able to uncover this pattern.

\section{Openness to Change}

Finally, we can ask how open to change the schema of interpretation is. In exploring this question, the schemas of contention theory makes contact with and enriches the existing studies of the temporalities of contention, allowing us to uncover a new aspect in the historical changes in contention (Jansen 2016; McAdam and Sewell 2001; Sewell 1996; Tarrow 1993b; Traugott 1993). While the studies have focused on the temporalities of the schema of action, we argue that the schema of interpretation may well adhere to a temporal rhythm that is both distinct from that of action and has internal differences of its own.

There is a disjuncture between changes to the schemas of action and interpretation. Tarrow (2011, pp. 111-18) gets at this in his discussion of the conventionalization of disruptive actions. However, 
because he does not distinguish the two schemas from each other, it remains unclear whether conventionalization describes the generalization of a tactic among actors or its lodgement in the schema of interpretation. Furthermore, the conflation maintains the assumption that the interpretation of contention follows the same rhythm as that of contentious action, albeit a beat or two behind. Contrary to this, we suggest that the schemas may be inscribed in different temporalities. This is mainly because of differences in the way that the schemas are encultured. Following Jansen's (2016) pragmatist approach, changes in the schema of action arise because situated contentious actors creatively respond to problem situations. These may both be small gradual adjustments (e.g., Tilly 1995) and eventful innovations (e.g., Sewell 1996), giving the schema of action a syncopated temporality characterized by stability and small changes that are sometimes punctured by radical innovations (McAdam and Sewell 2001, p. 113). This may differ from the schema of interpretation because the enculturation processes among the public are not primarily driven by problem-solving. The public do not actively reflect on or re-evaluate political tactics given the situation at hand. Rather, their enculturation process has more in common with the slow process that Lizardo (2017, pp. 92-3) associates with non-declarative personal culture. The schema of interpretation is acquired by learning about contention in school, hearing your parents discuss it, reading the newspapers, and seeing it dramatized in movies. It is deeper and less prone to change than the schema of action.

This might be especially true for the dimension of legibility. Whereas the ascription of legitimacy may be influenced by concerted framing, we suggest that ascriptions of legibility are not only more widely shared in the general public but also less open to change. As we note above, the illegibility of some tactics helps constitute the schema of interpretation. Rendering these legible is not simply a matter of readjustment but may involve rethinking the way that protest as such is understood. In studying changes to the public's interpretation of contention, it is therefore important to both disentangle it from changes to the schema of action and to distinguish between the dimensions of legitimacy and legibility.

\section{Limitations and Further Studies}

We have shown the value of theorizing and analyzing the schema of interpretation as a part of the schemas of contention. However, neither the analysis nor the discussion has exhausted the questions raised by the theory. Here, we briefly highlight three questions that are broached by our approach but not pursued here. 
First, we have focused on the schema of interpretation and not that of action. This was necessary in order to reveal the independent role of interpretation. However, we have not theorized or analyzed how the two schemas might be related once the assumption of a simple homology is done away with. Without a relation, contention would be impossible. We suggest that the relation can be theorized and analyzed through the concept of an emergent public culture (Lizardo 2017; Kurakin 2020). The discourses surrounding the acts of looting and property destruction following the murder of George Floyd provide an example. They partly emerged out of a discrepancy between the contentious actors' schema of action and a schema of interpretation in the public in which the actions were both illegitimate and illegible. At the same time, the discourses probably also worked back on the schemas. Discourses that highlighted the political nature of the acts might have made the actions of looting and property destruction more legible than they were previously, with knock-on effects for later protests involving looting and property destruction. Other discourses that distinguished between peaceful protests and violent looting might have cemented the illegibility of the tactics even further. Exploratory case studies of these and similar contentious events would help develop our understanding of the connection between schemas.

Second, because we mapped the schema of interpretation through survey studies, we specified their dimensions a priori. While this did allow us to explore our main theoretical contention concerning the existence of a schema of interpretation, it also meant that we had to keep the dimensions relatively broad and simple. There may be nuances to the two dimensions and alternative dimensions that we have not analyzed. In short, while we claim that the schema of interpretation exists, we do not claim that it exists only in two dimensions. Rather, we argue that the dimensions provide a useful theoretical model for the schema that is fuzzier than our model suggests. A complementary approach would be to analyze the schemas using Hunzaker and Valentino's (2019) concept-association approach, although this would focus less on the tactics themselves and more on the concepts that help constitute the notion of protest.

Third, we have sought to create an overall map of the individual tactics and have therefore not studied the relations between them. Because meaning is constituted relationally, this is a blind spot in our analyses. As we note above in the case of illegibility, there may differences between tactics that can only be uncovered by analyzing these relations. Interview studies focusing on these relations would complement our approach. In these ways, our analysis shows the fruitfulness of the schemas of contention theory but does not exhaust the range of questions that are raised by it. 


\section{Closing Remarks}

We argue that we need to theoretically and analytically distinguish the structuring of contentious actions from their interpretation. Our schemas of contention theory does this by focusing on the embodied principles that govern contention, distinguishing between a schema of action and a schema of interpretation. As the former has been studied extensively, we focus on the latter. Through two studies, one of which is representative of the US population, we show that the interpretation of contention is structured. That is, there is a schema of interpretation that is transposable across different contexts. Moreover, we can model this schema through the distinct dimensions of legitimacy and legibility. There is considerable variation in the degree to which contentious tactics are actually seen as both political and legitimate by the US public. This is strong empirical evidence for our theoretical contention that the schemas of action and interpretation need to be theorized and analyzed separately. It also opens up a new research agenda centered on these schemas. We suggest that future studies could build on it by analyzing the transposability of the schema of interpretation in other contexts, further investigate group differences in interpretation, and expand on the relations inside and between the schemas of interpretation and contention.

In following this line of research, we gain a new understanding of the structuring of contention and, therefore, the roads to social change. In liberal democracies in particular, contention works through the public (Alexander 2006; Koopmans 2004). This means that the structures that govern the public's interpretation of contentious actions both enable and constrain attempts to promote social change, as in the case of riots. This is not to say that only legitimate and legible tactics can bring about change. Sometimes calls for radical change are best expressed through tactics that challenge the extant schema of interpretation (Gillan 2020). Rather, the schema of interpretation is central to the potential for social change in contentious politics even when it is being challenged. If we wish to understand social change, we need to understand the schemas of interpretation through which individuals ascribe meaning to contention.

Analyzing the schemas of contention may also contribute directly to social change. In providing an empirical mapping of the schemas of contention, we can help contentious actors understand the public to whom they address themselves. This may aid their decisions on when to challenge the public's schema of interpretation and when to follow it. Furthermore, the schemas of contention theory makes possible an area of scholarly activism. In analyzing the public's interpretation of contention, we raise 


\section{Johan Gøtzsche-Astrup \& Oluf Gøtzsche-Astrup Aarhus University, DK Denmark}

the question of whether and how we can change it. For example, can we help the public ascribe political meaning, if not necessarily legitimacy, to tactics such as looting and property destruction that are employed by some of the most powerless groups in society? Our role might consist in both uncovering the schemas of contention and, through this uncovering, expanding them. 
Johan Gøtzsche-Astrup \& Oluf Gøtzsche-Astrup

Aarhus University, DK Denmark

\section{Literature}

Alexander, Jeffrey. 2006. The Civil Sphere. New York: Oxford University Press.

Andrews, Kenneth T., and Neal Caren. 2010. "Making the News: Movement Organizations, Media Attention, and the Public Agenda." American Sociological Review 75 (6): 841-66.

Andrews, Kenneth T., Kraig Beyerlein, and Tuneka Tucker Farnum. 2016. "The Legitimacy of Protest: Explaining White Southerners' Attitudes Toward the Civil Rights Movement." Social Forces 94 (3): 1021-44.

Benford, Robert. D., and David A. Snow. 2000. "Framing Processes and Social Movements: An Overview and Assessment." Annual Review of Sociology 26: 611-39.

Biggs, Michael. 2013. "How Repertoires Evolve: The Diffusion of Suicide Protest in the Twentieth Century.” Mobilization: An International Quarterly 18 (4): 407-28.

Body-Gendrot, Sophie. 2016. "Making Sense of French Urban Disorders in 2005." European Journal of Criminology 13 (5): 556-72.

Bourdieu, Pierre. 1990. The Logic of Practice. Stanford: Stanford University Press.

Doherty, Brian, and Graeme Hayes. 2019. “Tactics and Strategic Action.” Pp. 271-88 in The Wiley Blackwell Companion to Social Movements, edited by David A. Snow, Sarah Anne Soule, Hanspeter Kriesi, and Holly J. McCammon. Hoboken: Wiley Blackwell.

Ferree, Myra Marx, William Anthony Gamson, Jürgen Gerhards, and Dieter Rucht. 2002. Shaping Abortion Discourse: Democracy and the Public Sphere in Germany and the United States. Cambridge: Cambridge University Press.

Flesher-Fominaya, Cristina. 2015. "Debunking Spontaneity: Spain’s 15-M/Indignados as Autonomous Movement.” Social Movement Studies 14 (2): 142-63.

Giddens, Anthony. 1984. The Constitution of Society. Cambridge: Polity Press.

Gillan, Kevin. 2020. “Social Movements, Protest, and Practices of Social Change.” Pp. 301-18 in The Wiley Blackwell Companion to Sociology ( $2^{\text {nd }}$ edition), edited by George Ritzer and Wendy Wiedenhoft Murphy. Hoboken: Wiley Blackwell.

Goodwin, Jeff, and James M. Jasper. 1999. "Caught in a Winding, Snarling Vine: The Structural Bias of Political Process Theory.” Sociological Forum 14 (1): 27-54.

Hall, Robert L., Mark Rodeghier, and Bert Useem. 1986. "Effects of Education on Attitude to Protest." American Sociological Review 51 (4): 564-73.

Hunzaker, M. B. Fallin, and Lauren Valentino. 2019. "Mapping Cultural Schemas: From Theory to Method." American Sociological Review 84 (5): 950-81.

Jansen, Robert S. 2016. "Situated Political Innovation: Explaining the Historical Emergence of New Modes of Political Practice.” Theory and Society 45: 319-60. 
Jeffries, Vincent, Ralph H. Turner, and Richard T. Morris. 1971. "The Public Perception of the Watts Riot as Social Protest." American Sociological Review 36 (3): 443-51.

Koopmans, Ruud, and Susan Olzak. 2004. "Discursive Opportunities and the Evolution of RightWing Violence in Germany.” American Journal of Sociology 110 (1): 198-230.

Koopmans, Ruud. 2004. "Movements and Media: Selection Processes and Evolutionary Dynamics in the Public Sphere." Theory and Society 33: 367-391.

Kurakin, Dmitry. 2020. "Culture and Cognition: The Durkheimian Principle of Sui Generis Synthesis vs. Cognitive-based Models of Culture.” American Journal of Cultural Sociology 8: 63-89.

Lamont, Michelle, and Virág Molnár. 2002. “The Study of Boundaries in the Social Sciences.” $A n$ nual Review of Sociology 22: 167-95.

Larson, Jeff A. 2013. "Social Movements and Tactical Choice.” Sociology Compass 7: 866-79.

Lizardo, Omar. 2010. "Beyond the Antinomies of Structure: Levi-Strauss, Giddens, Bourdieu, and Sewell." Theory and Society 39: 651-88.

Lizardo, Omar. 2017. "Improving Cultural Analysis: Considering Personal Culture in its Declarative and Nondeclarative Modes." American Sociological Review 82 (1): 88-115.

McAdam, Doug, and Hilary Schaffer Boudet. 2012. Putting Social Movements in their Place: Explaining Opposition to Energy Projects in the United States, 2000-2005. New York: Cambridge University Press.

McAdam, Doug, and William H. Sewell, Jr. 2001. "It's About Time: Temporality in the Study of Social Movements and Revolutions." Pp. 89-125 in Silence and Voice in the Study of Contentious Politics, edited by Ronald R. Aminzade, Jack A. Goldstone, Doug McADam, Elizabeth J. Perry, William H. Sewell, Jr., Sidney Tarrow, and Charles Tilly. New York: Cambridge University Press.

McAdam, Doug, Sidney Tarrow, and Charles Tilly. 2001. Dynamics of Contention. Cambridge: Cambridge University Press.

McCurdy, Patrick, Anna Feigenbaum, and Fabian Frenzel. 2016. "Protest Camps and Repertoires of Contention." Social Movement Studies 15 (1): 97-104.

Olsen, Marvin E. 1968. "Perceived Legitimacy of Social Protest Actions.” Social Problems 15 (3): 297310.

Park, Soon Seok, and Rachel L. Einwohner. 2019. "Becoming a Movement Society? Patterns in the Public Acceptance of Protest, 1985-2006.” Sociological Focus 52 (3): 186-200.

Ring-Ramirez, Misty, Reynolds-Stenson, Heidi, and Earl, Jennifer. 2014. "Culturally Constrained Contention: Mapping the Meaning Structure of the Repertoire of Contention.” Mobilization 19 (4): 405-19.

Robinson, Jonathan, Cheskie Rosenzweig, Aaron J. Moss, and Leib Litman. 2019. "Tapped out or Barely Tapped? Recommendations for How to Harness the Vast and Largely Unused Potential of the Mechanical Turk Participant Pool." PloS One 14 (12), e0226394. https://doi.org/10.1371/journal.pone.0226394 
Rodeghier, Mark, Robert L. Hall, and Bert Useem. 1991. "How Education Affects Attitude to Protest: A Further Test.” The Sociological Quarterly 32 (2): 277-88.

Seguin, Charles. 2015. "Cascades of Coverage: Dynamics of Media Attention to Social Movement Organizations.” Social Forces 94 (3): 997-1020.

Sewell, William H., Jr. 1996. "Historical Events as Transformations of Structures: Inventing Revolution at the Bastille." Theory and Society 25: 841-81.

Sewell, William H., Jr. 2005. Logics of History. Chicago: University of Chicago Press.

Simpson, Brent, Robb Willer, and Matthew Feinberg. 2018. "Does Violent Protest Backfire? Testing a Theory of Public Reactions to Activist Violence." Socious: Sociological Research for a Dynamic World 4: $1-14$.

Snow, David A., Rens Vliegenthart, and Pauline Ketelaars. 2019. “The Framing Perspective on Social Movements: Its Conceptual Roots and Architecture." Pp. 392-410 in The Wiley Blackwell Companion to Social Movements, edited by David A. Snow, Sarah Anne Soule, Hanspeter Kriesi, and Holly J. McCammon. Hoboken: Wiley Blackwell.

Swidler, Ann. 1986. "Culture in Action: Symbols and Strategies." American Sociological Review 51 (2): 273-86.

Tarrow, Sidney. 1993a. "Modular Collective Action and the Rise of the Social Movement: Why the French Revolution Was Not Enough.” Politics \& Society 21 (1): 69-90.

Tarrow, Sidney. 1993b. "Cycles of Collective Action: Between Moments of Madness and the Repertoire of Contention.” Social Science History 17 (2): 281-307.

Tarrow, Sidney. 2011. Power in Movement: Social Movements and Contentious Politics. Cambridge: Cambridge University Press.

Taylor, Charles. 1971. "Interpretation and the Sciences of Man." The Review of Metaphysics 25 (1): 351.

Taylor, Verta, Katrina Kimport, Nella Van Dyke, and Ellen Ann Andersen. 2009. "Culture and Mobilization: Tactical Repertoires, Same-sex Weddings, and the Impact on Gay Activism." American Sociological Review 74 (6): 865-90.

Tilly, Charles. 1977. "Getting it together in Burgundy, 1676-1975." Theory and Society 4 (4): 479-504.

Tilly, Charles. 1995. Popular Contention in Great Britain 1750-1834. Boulder: Paradigm Publishers.

Tilly, Charles. 2008. Contentious Performances. Cambridge: Cambridge University Press.

Traugott, Mark. 1993. "Barricades as Repertoire: Continuities and Discontinuities in the History of French Contention." Social Science History 17 (2): 309-23.

Turner, Ralph H. 1969. “The Public Perception of Protest.” American Sociological Review 34 (6): 81531. 
Johan Gøtzsche-Astrup \& Oluf Gøtzsche-Astrup

Aarhus University, DK Denmark

Vasi, Ion Bogdan, Edward T. Walker, John S. Johnson, and Hui Fen Tan. 2015. "No Fracking Way!” Documentary Film, Discursive Opportunity, and Local Opposition against Hydraulic Fracturing in the United States, 2010 to 2013. American Sociological Review 80 (5): 934-59.

Vliegenthart, Rens, Stefaan Walgrave, Ruud Wouters, Swen Hutter, Will Jennings, Roy Gava, Anke Tresch, Frédéric Varone, Emiliano Grossman, Christian Breunig, Sylvain Brouard, and Laura Chaques-Bonafont. 2016. The Media as a Dual Mediator of the Political Agenda-Setting Effect of Protest. A Longitudinal Study in Six Western European Countries. Social Forces 95 (2): 837-59.

Wada, Takeshi. 2012. "Modularity and Transferability of Repertoires of Contention." Social Problems 59 (4): 544-71.

Wada, Takeshi. 2016. "Rigidity and Flexibility of Repertoires of Contention." Mobilization: An International Quarterly 21 (4): 449-68.

Wang, Dan J., and Sarah Anne Soule. 2016. "Tactical Innovation in Social Movements: The Effects of Peripheral and Multi-issue Protest." American Sociological Review 81 (3): 517-48.

Wei, Wei. 2015. "Street, Behaviour, Art: Advocating Gender Rights and the Innovation of a Social Movement Repertoire." Chinese Journal of Sociology 1 (2): 279-304.

Wood, Michael Lee, Dustin S. Stoltz, Justin Van Ness, and Marshall A. Taylor. 2018. "Schemas and Frames." Sociological Theory 36 (3): 244-61.

Wouters, Ruud. 2019. “The Persuasive Power of Protest: How Protest Wins Public Support.” Social Forces 98 (1): 403-26.

Yates, Luke. 2015. "Rethinking Prefiguration: Alternatives, Micropolitics and Goals in Social Movements." Social Movement Studies 14 (1): 1-21.

Zlobina, Anna, and Alejandro Gonzalez Vazquez. 2018. "What Is the Right Way to Protest? On the Process of Justification of Protest, and Its Relationship to the Propensity to Participate in Different Types of Protest." Social Movement Studies 17 (2): 234-50. 


\section{Tables and figures}

Table 1. Tactics Included in This Study

1. Ringing bells

2. Lighting candles

3. Putting up posters

4. Dancing

5. Having a public discussion

6. Going on a hunger strike

7. Setting off fireworks

8. Sending out leaflets

9. Sitting in meditation

10. Collecting signatures for a petition

11. Holding signs in public

12. Marching in the streets

13. Selling items marking the shooting

14. Holding a speech

15. Shouting slogans

16. Holding a silent vigil

17. Worshipping

18. Laying down wreaths

19. Holding a press conference

20. Hosting an art exhibition (e.g., showing films or exhibiting photos)

21. Recruiting members to organizations combating shootings

22. Lobbying politicians

23. Conducting an opinion poll

24. Passing torches

25. Meeting with politicians

26. Distributing goods (e.g., food, clothes)

27. Doing a sit-in

28. Physically attacking someone

29. Holding an art performance (e.g., a play, a musical, singing, or drumming)

30. Posting your opinion on the shooting on social media

31. Verbally attacking someone

32. Blockading roads or buildings

33. Making loud noises in public

34. Taking over a building

35. Looting (e.g., stores, homes)

36. Damaging property

37. Kidnapping and holding people hostage

38. Disrupting meetings

39. Conducting a walkout

40. Filing a lawsuit

41. Sharing news coverage of the shooting on social media 
Table 2. Means and Standard Deviations for Tactics for Legitimacy and Legibility, Study 2 ( $N=$ 1033)

\begin{tabular}{|c|c|c|c|}
\hline & Legitimacy & Legibility & Difference \\
\hline Ringing bells & $58.4(34.0)$ & $38.2(33.8)$ & $20.3^{* * *}$ \\
\hline Lighting candles & $79.4(27.5)$ & $43.9(36.7)$ & $35.5^{* * *}$ \\
\hline Putting up posters & $70.1(30.0)$ & $61.7(30.9)$ & $8.38^{* * *}$ \\
\hline Dancing & $41.5(37.1)$ & $24.7(29.3)$ & $16.8^{* * *}$ \\
\hline Having a public discussion & $78.3(25.8)$ & $62.6(31.9)$ & $15.7^{* * *}$ \\
\hline Going on a hunger strike & $42.2(34.4)$ & $53.3(35.8)$ & $-11.0^{* * *}$ \\
\hline Setting off fireworks & $32.4(33.0)$ & $31.1(31.8)$ & 1.26 \\
\hline Sending out leaflets & $69.1(29.9)$ & $58.9(31.8)$ & $10.2^{* * *}$ \\
\hline Sitting in meditation & $72.7(30.2)$ & $41.5(35.5)$ & $31.3^{* * *}$ \\
\hline $\begin{array}{l}\text { Collecting signatures for a peti- } \\
\text { tion }\end{array}$ & $76.2(27.6)$ & $74.0(27.8)$ & $2.24 *$ \\
\hline Holding signs in public & $68.9(29.8)$ & $70.7(28.0)$ & -1.85 \\
\hline Marching in the streets & $59.9(33.4)$ & $72.2(30.9)$ & $-12.4 * * *$ \\
\hline $\begin{array}{l}\text { Selling items marking the shoot- } \\
\text { ing }\end{array}$ & $32.2(33.3)$ & $38.4(34.8)$ & $-6.21 * * *$ \\
\hline Holding a speech & $74.3(27.1)$ & $66.5(30.1)$ & $7.78^{* * *}$ \\
\hline Shouting slogans & $45.0(34.4)$ & $60.4(34.3)$ & $-15.4 * * *$ \\
\hline Holding a silent vigil & $80.9(26.8)$ & $49.6(36.8)$ & $31.3^{* * *}$ \\
\hline Worshipping & $72.1(31.9)$ & $36.1(35.4)$ & $36.0^{* * *}$ \\
\hline Laying down wreaths & $77.2(28.7)$ & $41.6(35.5)$ & $35.6^{* * *}$ \\
\hline Holding a press conference & $75.1(27.0)$ & $63.2(31.6)$ & $11.9^{* * *}$ \\
\hline $\begin{array}{l}\text { Hosting an art exhibition (e.g., } \\
\text { showing films or exhibiting } \\
\text { photos) }\end{array}$ & $56.3(36.1)$ & $37.6(33.2)$ & $18.7^{* * *}$ \\
\hline $\begin{array}{l}\text { Recruiting members to organi- } \\
\text { zations combating shootings }\end{array}$ & $61.3(35.2)$ & $60.3(34.1)$ & 0.99 \\
\hline Lobbying politicians & $65.7(32.3)$ & $70.8(30.3)$ & $-5.14 * * *$ \\
\hline Conducting an opinion poll & $69.0(31.2)$ & $57.2(32.8)$ & $11.9^{* * *}$ \\
\hline Passing torches & $52.1(34.3)$ & $44.5(33.1)$ & $7.57^{* * *}$ \\
\hline Meeting with politicians & $75.2(27.6)$ & $68.2(31.3)$ & $7.06^{* * *}$ \\
\hline $\begin{array}{l}\text { Distributing goods (e.g., food, } \\
\text { clothes) }\end{array}$ & $68.5(33.3)$ & $36.0(34.4)$ & $32.5^{* * *}$ \\
\hline Doing a sit-in & $61.3(32.6)$ & $65.1(32.3)$ & $-3.88^{* * *}$ \\
\hline Physically attacking someone & $15.9(27.8)$ & $32.8(36.4)$ & $-16.9 * * *$ \\
\hline $\begin{array}{l}\text { Holding an art performance } \\
\text { (e.g., a play, a musical, singing or } \\
\text { drumming) }\end{array}$ & $58.7(35.6)$ & $34.9(32.7)$ & $23.8^{* * *}$ \\
\hline $\begin{array}{l}\text { Posting your opinion on the } \\
\text { shooting on social media }\end{array}$ & $67(31.3 .0)$ & $52.5(33.1)$ & $14.5^{* * *}$ \\
\hline Verbally attacking someone & $21.3(28.6)$ & $39.3(35.8)$ & $-17.9 * * *$ \\
\hline Blockading roads or buildings & $25.6(32.0)$ & $53.2(38.5)$ & $-27.6^{* * *}$ \\
\hline Making loud noises in public & $32.2(32.4)$ & $44.3(34.7)$ & $-12.0^{* * *}$ \\
\hline Taking over a building & $20.2(29.6)$ & $50.1(38.9)$ & $-30.0 * * *$ \\
\hline Looting (e.g., stores, homes) & $15.2(27.9)$ & $35.0(38.1)$ & $-19.8^{* * *}$ \\
\hline
\end{tabular}


Johan Gøtzsche-Astrup \& Oluf Gøtzsche-Astrup Aarhus University, DK Denmark

\begin{tabular}{|c|c|c|c|}
\hline Damaging property & $15.4(27.3)$ & $38.9(38.0)$ & $-23.5^{* * *}$ \\
\hline $\begin{array}{l}\text { Kidnapping and holding people } \\
\text { hostage }\end{array}$ & $14.4(28.0)$ & $37.7(39.5)$ & $-23.3^{* * *}$ \\
\hline Disrupting meetings & $28.0(31.5)$ & $52.7(36.4)$ & $-24.7 * * *$ \\
\hline Conducting a walkout & $52.7(34.8)$ & $65.4(33.0)$ & $-12.6^{* * * *}$ \\
\hline Filing a lawsuit & $67.0(30.8)$ & $55.4(33.9)$ & $11.6^{* * *}$ \\
\hline Sharing news coverage of the & $68.4(29.9)$ & $55.0(33.4)$ & $13.4^{* * *}$ \\
\hline
\end{tabular}
shooting on social media

Note: Results from two-sample $t$-tests. Standard deviations reported in parentheses. Significance levels: $*<.05, * *<.01, * * *<.001$. 
Figure 1. Means for Legibility and Legitimacy, Nat. Rep. Study $(N=1033)$

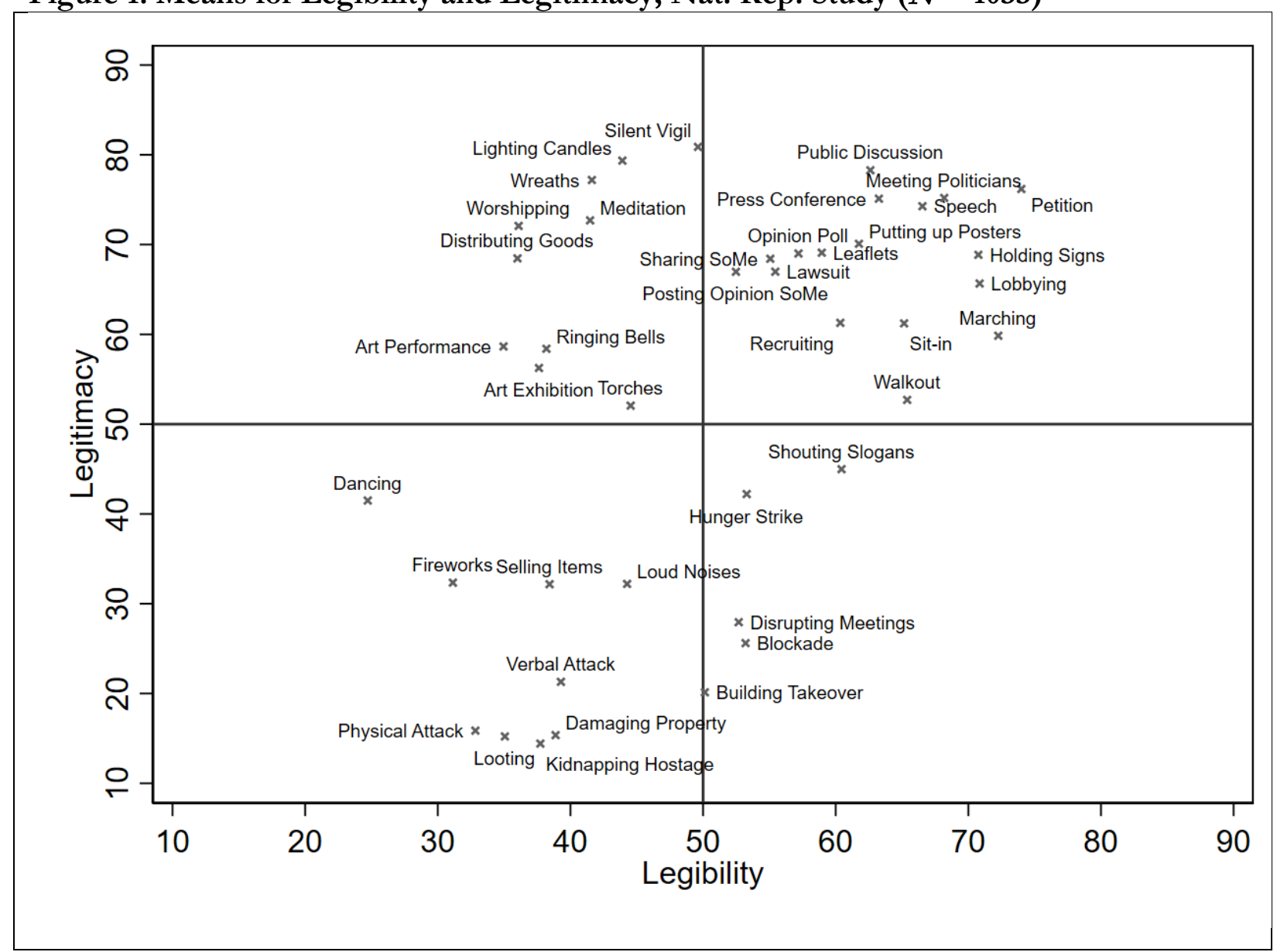


Figure 2. Graph of the 14 Tactics Differing Significantly in at Least One Dimension between the Two Contexts

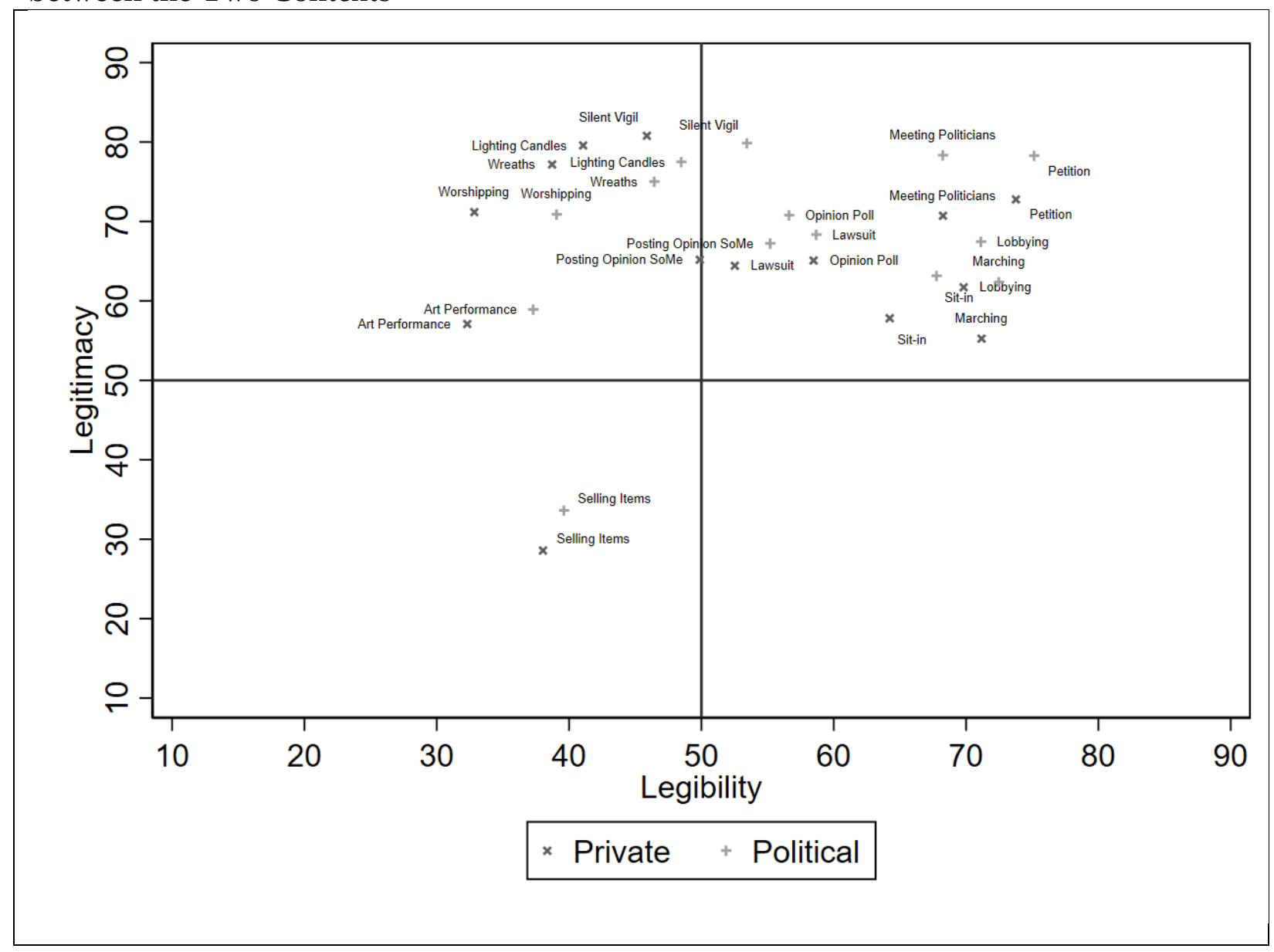

